\title{
Uniaxial and Radial Anisotropy Models for Finite-Volume Maxwellian Absorber
}

\author{
Krishnaswamy Sankaran, Student Member, IEEE, Christophe Fumeaux, Member, IEEE, and \\ Rüdiger Vahldieck, Fellow, IEEE
}

\begin{abstract}
The uniaxial finite-volume Maxwellian absorber used as a perfectly matched layer is extended to incorporate radial anisotropy for modeling cylindrical geometries. Theoretical background and practical applications of both uniaxial and radial absorber models are presented. Both these models employ spatially and temporally co-located electromagnetic field quantities in an unstructured mesh. The uniaxial Maxwellian absorber model is tested for a truncated waveguide problem. The influence of absorber thickness and material loss parameter on the performance of the model is analyzed. Numerical reflection coefficients down to $-60 \mathrm{~dB}$ are achieved for fine mesh discretization with approximately 20 points per wavelength confirming the convergence of numerical results. As an extension of the technique, a radially anisotropic absorber model is tested for cylindrical mesh truncation using a representative problem involving two different test scenarios. Results are compared with an existing technique commonly used in finite-volume time-domain simulations, demonstrating substantial reduction in numerical error due to cylindrical mesh truncation.
\end{abstract}

Index Terms-Computational electromagnetics (CEM), finite volume time domain (FVTD), Maxwellian absorber, perfectly matched layer (PML), radial absorber.

\section{INTRODUCTION}

A FINITE-VOLUME-BASED uniaxial Maxwellian absorber was introduced by the authors in [1] with an application to waveguide truncation problem. For scattering and antenna applications, this uniaxial model suffers from inaccuracies originating from the corner regions, which are the major sources of numerical reflections (inaccuracies) in the computed solution. Therefore, to avoid the corner regions, a radial absorber model is introduced in this paper. This radial Maxwellian absorber model uses a novel approach to achieve anisotropy in the radial direction and can be efficiently used in applications involving cylindrical or spherical geometries. The theory of the radial absorber is developed as an extension to the uniaxial model, and supporting numerical experiments are presented. The present finite-volume time-domain (FVTD) approach naturally adapts the model for application on unstructured meshes and it is based on the modified Lorentz material response of lossy dielectric media, as proposed in [2]-[4]. As compared to the convolutional perfectly matched layer (PML) discussed in [5], there is no need for performing a convolution

Manuscript received April 6, 2006; revised August 9, 2006. This work was supported under ETH Research Grant TH-38/04-1.

The authors are with the Laboratory for Electromagnetic Fields and Microwave Electronics, Swiss Federal Institute of Technology Zürich, Zürich CH-8092, Switzerland (e-mail: krishna@ifh.ee.ethz.ch; fumeaux@ifh.ee.ethz.ch; vahldieck@ifh.ee.ethz.ch).

Digital Object Identifier 10.1109/TMTT.2006.885577 operation during each time step. Furthermore, there is no need for unphysical field-splitting, as in the case of Bérenger's split PMLs [6], [7] and, hence, the model satisfies Maxwell equations both inside and outside the absorber region. Unsplit-PML models have been previously studied. mainly in the framework of the spatially and temporally staggered finite-difference time-domain (FDTD) method or for the finite-element time-domain (FETD) method. The standard staircase approximation near domain boundaries impose constraints on the flexibility and accuracy of the FDTD method. This creates a strong motivation for the development of the FVTD method, which can handle unstructured meshes and additionally enables spatially and temporally co-located field storage. Using curvilinear PML models, the corner regions can be avoided in the computational domain. The curvilinear PML models reported in [8]-[11] were adapted mainly for FDTD and FETD methods, which uses update equations represented in cylindrical coordinate. The new finite-volume radial absorber model introduced in this paper is based on a different approach, which capitalizes on the capability of the FVTD method to handle unstructured mesh to its full advantage. Hence, the modeling of cylindrical geometry becomes possible without the need for coordinate transformation, as in the case of FDTD simulations. Achieving radial anisotropy using Cartesian domain formulation involves rotational transformation from locally uniaxial to globally radial behavior of the material loss parameter.

This paper is organized as follows. In Section II, a brief introduction of the FVTD method is presented for modeling the modified Lorentz media. In Section III, a two-dimensional (2-D) FVTD formulation of the modified lossy Lorentz media is discussed and the relevant update equations are presented. A practical application of the uniaxial finite-volume absorber is demonstrated for the waveguide truncation problem. Some numerical implementation aspects of the uniaxial Maxwellian absorber are discussed, emphasizing the optimal choice of absorber thickness and material loss parameter. The theory of radial finite-volume Maxwellian absorber is introduced in Section IV. The concept of radial anisotropy is explained using a locally rotated uniaxial absorber model. As a validation, the radial absorber is tested for sample problems with cylindrical geometry. Finally, the performance of the radial absorber is compared with an existing mesh truncation technique.

\section{FVTD Model of Modified LORENTZ Media}

In the context of FVTD modeling, the continuous time-dependent Maxwell equations are discretized both in space and time using finite samples. Each spatial-sample $\Omega_{i}$ is called a control volume or cell and the temporal-sample $\Delta t$ corresponds 
to the update time step. Depending on the method of storing and updating the field quantities, different FVTD schemes are possible. For a detailed description of the FVTD method, please refer to [12]-[15]. The absorber model presented here provides simple and consistent co-located spatial and temporal field variations. In the following analysis, a 2-D transverse electric $\left(\mathrm{TE}_{z}\right)$ model with the electric field along the $z$-axis $\left(E_{z}\right)$ and the magnetic field in the $x y$-plane $\left(H_{x}\right.$ and $\left.H_{y}\right)$ is employed. This 2-D model is tested on computational domains meshed with unstructured (conformal) triangular cells. Using the divergence theorem, the FVTD update equations inside each cell of the dielectric medium is cast in the form [14]

$$
\alpha_{0} \partial_{t} \boldsymbol{U}=-\frac{1}{\left|V_{i}\right|} \sum_{k=1}^{f}\left(\mathcal{F}_{\boldsymbol{U}_{k}} \cdot \boldsymbol{n}_{k}\left|S_{k}\right|\right)-\alpha_{0} \partial_{t} \boldsymbol{Q}
$$

where $\boldsymbol{U}=\left[H_{x}, H_{y}, E_{z}\right]^{T}$ denotes the electromagnetic (EM) field vector with the superscript $T$ representing the matrix transpose. Each $i$ th polyhedral cell is made of $f$ faces and has a control-volume $\left|V_{i}\right|$ (corresponding to an area in the 2-D model). Each $k$ th face has an area of $\left|S_{k}\right|$ (length in the 2-D model) and a unit outward-normal $\boldsymbol{n}_{k}$. The factor $\alpha_{0}$ takes the value of free-space permeability $\left(\mu_{0}\right)$ and permittivity $\left(\varepsilon_{0}\right)$ for the magnetic and electric field update equations, respectively. The vector $\boldsymbol{Q}=\left[M_{x}, M_{y}, P_{z} / \varepsilon_{0}\right]^{T}$ represents the components of magnetization $\boldsymbol{M}$ and polarization $\boldsymbol{P}$ fields inside the dielectric medium. The factor $\mathcal{F}_{\boldsymbol{U}_{k}} \cdot \boldsymbol{n}_{k}$ is called the flux function in the FVTD nomenclature and plays a crucial role in information exchange between adjacent cells.

\section{UNIAXIAL FINITE-VOLUME MAXWELLIAN ABSORBER}

The polarization $\boldsymbol{P}$ and magnetization $\boldsymbol{M}$ field vectors of the modified lossy Lorentz media were previously used to model FDTD-based absorbing boundary conditions (ABCs) [2], [3]. These auxiliary field quantities, when included in the FVTD formulation, result in computational overhead due to additional update equations involving flux terms. In this paper, a modified approach, which reduces the computational overhead in the FVTD method, is employed to model the Maxwellian absorber on a 2-D unstructured triangular grid. The $\mathrm{TE}_{z}$ model of the modified (time-derivative) Lorentz material yields the magnetization and polarization equations as follows [4]:

$$
\left(\partial_{t}^{2}+\Gamma \partial_{t}+\omega_{0}^{2}\right) \boldsymbol{Q}=(\omega_{0}^{2} \chi_{\alpha}+\overbrace{\omega_{0} \chi_{\beta} \partial_{t}}^{\text {additional term }}) \boldsymbol{U}
$$

where the material resonance-frequency is denoted as $\omega_{0}$ and the resonance bandwidth is given by $\Gamma$. The factor $\chi_{\alpha}$ relates $\boldsymbol{P}$ and $\boldsymbol{M}$ to $\boldsymbol{E}$ and $\boldsymbol{H}$, respectively. Similarly, the term $\chi_{\beta}$ couples $\boldsymbol{P}$ and $\boldsymbol{M}$ with time histories of $\boldsymbol{E}$ or $\boldsymbol{H}$, respectively. In particular, the factor $\chi_{\beta}$ plays a crucial role for numerical modeling and will be the subject of a detailed analysis in Section III-A. For a perfectly matched interface, a uniaxial absorber along the $x$-axis is considered. The frequency-domain electric and magnetic susceptibilities of the modified Lorentz media $\chi_{\omega}^{m}$ then results in the relative permittivity and permeability tensors given by

$$
\left(\begin{array}{ccc}
\left(1+\chi_{\omega}^{m}\right)^{-1} & 0 & 0 \\
0 & 1+\chi_{\omega}^{m} & 0 \\
0 & 0 & 1+\chi_{\omega}^{m}
\end{array}\right)
$$

where the superscript $m$ denotes the modified Lorentz model due to the additional time-derivative term in (2). The frequencydomain electric and magnetic susceptibilities are directly obtained from (2) using Fourier transformation as follows:

$$
\chi_{\omega}^{m}=\frac{\omega_{0}^{2}\left[\chi_{\alpha}-i\left(\omega / \omega_{0}\right) \chi_{\beta}\right]}{\omega_{0}^{2}-\omega^{2}-i \Gamma \omega}
$$

where subscript $\omega$ represents the frequency-domain value with $\omega$ corresponding to the center frequency of the incident EM excitation. As proposed in [2], the modified Lorentz model behaves like a uniaxial absorber if it satisfies the following constraints.

- $\omega \gg \omega_{0}$ and $\Gamma \ll \omega_{0}$ : this implies that the frequency of operation is much higher than the narrow resonance band of the material.

- $\chi_{\alpha} \ll 1$ : this avoids dispersion inside the absorber.

Under these constraints, (3) and (4) results in the modified polarization and magnetization field equations written as

$$
\begin{aligned}
\partial_{t}^{2} M_{x}+\zeta \partial_{t} M_{x} & =-\zeta \partial_{t} H_{x} \\
\partial_{t} M_{y} & =\zeta H_{y} \\
\partial_{t} P_{z} & =\varepsilon_{0} \zeta E_{z}
\end{aligned}
$$

The factor $\zeta$ is the material loss parameter inside the Maxwellian absorber, which is given by $\zeta=\chi_{\beta} \omega_{0}$. Using these constraints in (3) and (4), the modified polarization and magnetization fields are used to derive the field update equations, as given in [1]

$$
\begin{aligned}
& \partial_{t} H_{x}=-\frac{1}{\mu_{0}\left|V_{i}\right|} \sum_{k=1}^{f}\left(\mathcal{F}_{H_{x k}} \cdot \boldsymbol{n}_{k}\left|S_{k}\right|\right)+\zeta H_{x}-G_{x} \\
& \partial_{t} H_{y}=-\frac{1}{\mu_{0}\left|V_{i}\right|} \sum_{k=1}^{f}\left(\mathcal{F}_{H_{y k}} \cdot \boldsymbol{n}_{k}\left|S_{k}\right|\right)-\zeta H_{y} \\
& \partial_{t} E_{z}=-\frac{1}{\varepsilon_{0}\left|V_{i}\right|} \sum_{k=1}^{f}\left(\mathcal{F}_{E_{z k}} \cdot \boldsymbol{n}_{k}\left|S_{k}\right|\right)-\zeta E_{z} \\
& \partial_{t} G_{x}=-\zeta G_{x}+\zeta^{2} H_{x}
\end{aligned}
$$

where each component of $\mathcal{F}_{\boldsymbol{U}_{k}}$ explicitly depends on all the field quantities in $\boldsymbol{U}_{k}$. The factors of polarization and magnetization currents in (9) and (10) have the form of lossy conductivity terms inside the Maxwellian absorber. It can be noticed from the above system of equations that there are three standard EM field equations in $\boldsymbol{U}(8)-(10)$ and an additional update equation for the scalar field $G_{x}$, as in [16]. In contrast to [4], the update equation of the fourth field $\left(G_{x}\right)$ is an ordinary differential equation (ODE) in time and, hence, there is no substantial increase in the computational effort. Equations (8)-(11) constitute the final set of equations required to update the electric and 


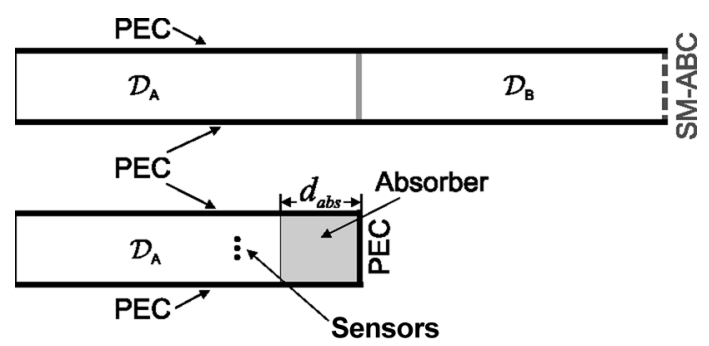

Fig. 1. Models for calculating reflection coefficient. (top) Reference model. (bottom) Test model. From [1]

magnetic field quantities. The whole computational domain $\mathcal{O}$ is subdivided into two parts, namely, the main computational domain $\mathcal{O}_{\text {main }}$ and the FVTD Maxwellian absorber domain $\mathcal{O}_{\text {abs }}$. The final update equations are similar in both domains with the additional requirement that $\zeta=0$ inside $\mathcal{O}_{\text {main }}$ and $\zeta \geq 0$ inside $\mathcal{O}_{\text {abs. }}$. This highlights an important advantage of Maxwellian absorber as compared to the split PML [6]. A parabolic profile is chosen for the variation of $\zeta$ inside $\mathcal{O}_{\text {abs }}$, increasing from 0 at the free-space absorber (FS-A) interface to a maximum value $\zeta_{0}$ at the absorber truncation boundary. In numerical experiments reported before in [2] and [6], this choice of profile variation resulted in optimal performance. Furthermore, the tolerance range of the parabolic profile was found to be better compared to other options [17].

\section{A. Practical Application: Waveguide Truncation Problem}

The performance of the FVTD Maxwellian absorber on unstructured mesh is tested using a 2-D parallel-plate waveguide problem. The waveguide is assumed to have infinite symmetry along the transverse $z$-axis. The wave propagation direction is towards the $+x$-axis. A perfect electrically conducting (PEC) boundary condition (BC) is forced on the two sides of the waveguide. The proposed FVTD Maxwellian absorber is used to truncate the waveguide perpendicularly to the $x$-axis. In order to verify the practical applicability of the FVTD Maxwellian absorber under the constraint of limited computational resource, the thickness of the absorber is first fixed to $d_{\mathrm{abs}}=1 \lambda$, where $\lambda$ corresponds to the wavelength at the center frequency $\omega$ of the EM excitation (pulse or harmonic). A triangular spatial discretization with cell edge dimensions of $\approx \lambda / 12$ is used for the results presented here.

In order to quantify the performance, the numerical reflection coefficient from the FVTD Maxwellian absorber is calculated for various angles of wave incidence. Considering the plane wave decomposition model of a waveguide mode, changing the width of the waveguide is equivalent to changing the angle of incidence with respect to the FS-A interface. For each angle of incidence, two models are built, namely, the reference and test models. The reference model is divided into two parts, one $\left(\mathcal{D}_{A}\right)$ with exactly the same domain cells as in the test model, and the other $\left(\mathcal{D}_{B}\right)$, an extension, which is truncated by a first-order Silver-Mueller ABC (SM-ABC), as shown in Fig. 1. The numerical reflection is computed by subtracting the reference field values from those of the test model. The model in Fig. 1 is used to study the reflection coefficient at different angles of incidence using a first-order TE-mode excitation. The analysis is carried

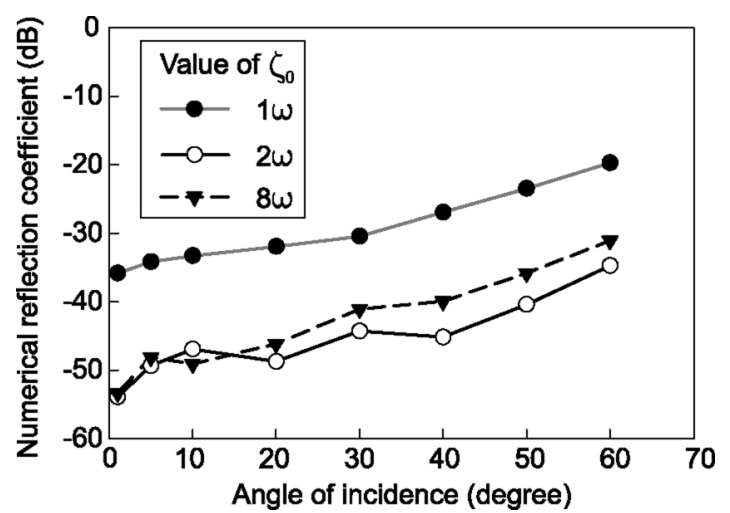

Fig. 2. Angular response of the FVTD Maxwellian absorber for different material loss parameter $\zeta_{0}$. From [1].

out over a range of incidence angles from near normal to $60^{\circ}$, which is of practical interest. The numerical reflection coefficient is calculated for different values of maximum material loss parameter $\zeta_{0}$ and the results are shown in Fig. 2 for $\omega, 2 \omega$, and $8 \omega$. For lower values of $\zeta_{0}$, e.g., $\zeta_{0} \approx \omega$, a relatively high numerical reflection coefficient exists, which is primarily originating from the absorber truncating (PEC) BC. On the other hand, for higher values, i.e., $\zeta_{0} \approx 8 \omega$, the numerical reflection comes from the FS-A interface. In fact, the later numerical reflection is mainly due to the spatial discretization errors. For most of the simulations, a value of $\zeta_{0} \approx 2 \omega$ resulted in minimum numerical reflection.

\section{B. Absorber Thickness Versus Performance}

The thickness of the absorber has a strong influence on its performance. In addition, the material loss parameter controls the damping behavior inside the absorber. In the case of split-field Bérenger PML [6], [17], for a desired (theoretical) reflection coefficient and a given thickness and loss profile inside the absorber, one could easily find the required value of maximum loss inside the PML. On the contrary, for the Maxwellian absorber model, there exists no straightforward way of deriving the maximum loss parameter required for a specified (theoretical) reflection coefficient considering the given constraints on the absorber thickness and loss profile. Hence, the study of combined effects of material loss parameter and absorber thickness on performance is a vital information for practical application of the absorber. With this objective, a model with triangular spatial discretization with edge dimension $\approx \lambda / 20$ is tested for various absorber thicknesses $d_{\text {abs }}$ ranging from $0.05 \lambda$ to $1.8 \lambda$. The numerical reflection coefficients are computed for three different values of material loss parameter $\zeta_{0}$ defined as before. The results of the analysis are shown in Figs. 3-5. As noticed in Fig. 3, the overall performance of the model is very low for $\zeta_{0}=1 \omega$. Especially, for absorber thickness $d_{\mathrm{abs}} \leq 1 \lambda$, this choice $\left(\zeta_{0}=1 \omega\right)$ results in high reflection. Therefore, for acceptable performance, a large absorber thickness is required, which increases the computational effort. The reason for this high reflection is a direct consequence of the very low damping present inside the absorber domain. Hence, in spite of damping of the wave on its forward and return paths, a significant part of the power is reflected back into the main computational domain 


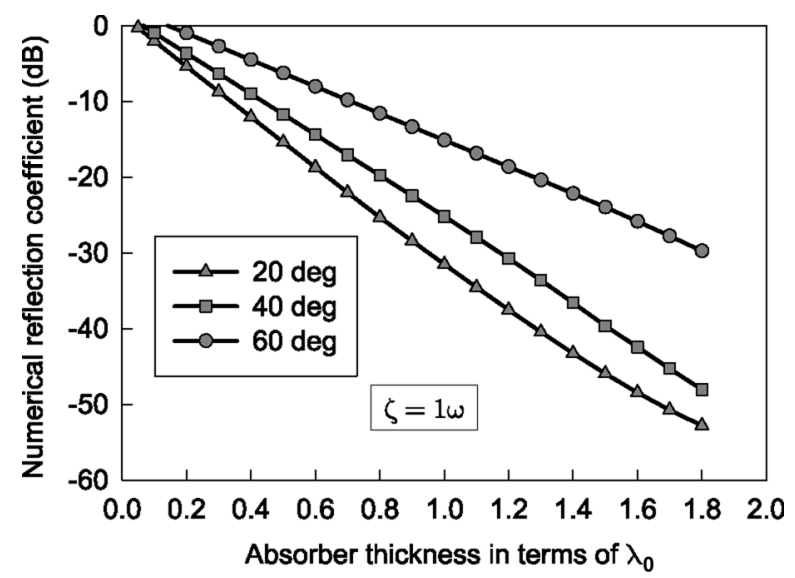

Fig. 3. Effect of absorber thickness on its performance at three different angles of incidence for material loss parameter $\zeta_{0}=1 \omega$.

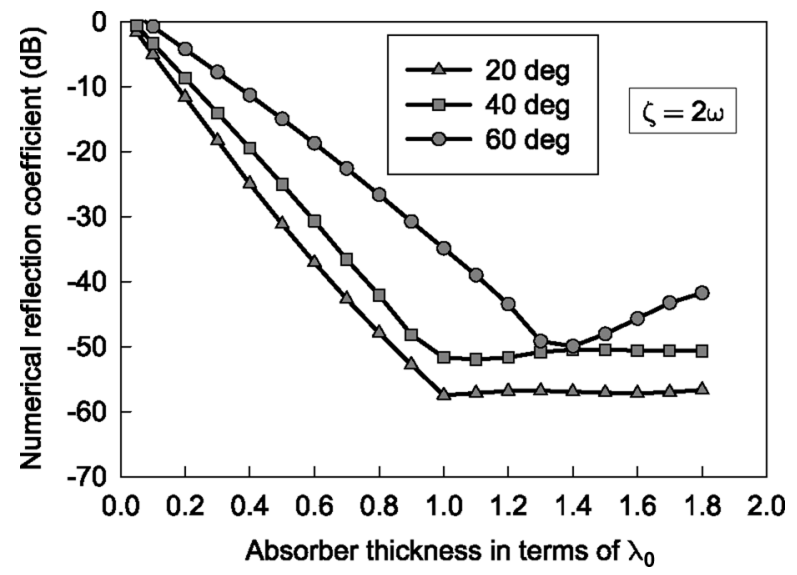

Fig. 4. Effect of absorber thickness on its performance at three different angles of incidence for material loss parameter $\zeta_{0}=2 \omega$.

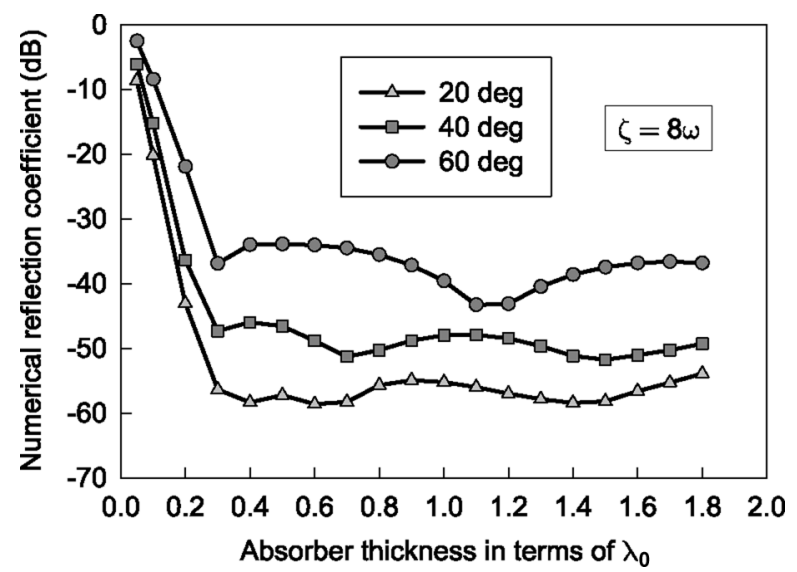

Fig. 5. Effect of absorber thickness on its performance at three different angles of incidence for material loss parameter $\zeta_{0}=8 \omega$.

from the truncating PEC of the absorber. When the value of $\zeta_{0}$ is increased to $2 \omega$ and $8 \omega$, the damping inside the absorber is substantially increased, resulting in improvement in the performance shown in Figs. 4 and 5. The oscillations noticed in the numerical reflection coefficient value in Fig. 5 correspond to constructive and destructive interferences between waves reflected at the FS-A interface and at the truncating PEC. For low values

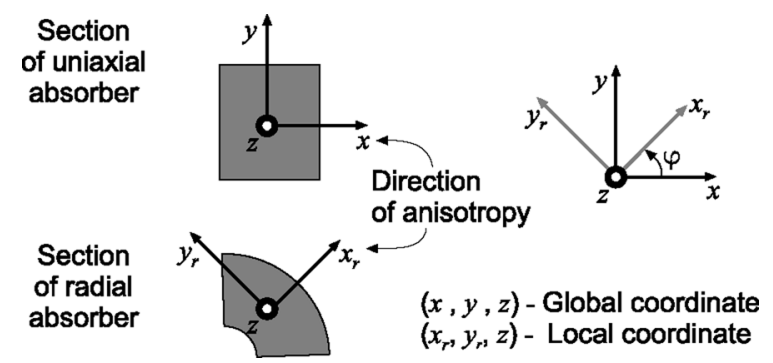

Fig. 6. Graphical illustration of radial anisotropy using coordinate transformation by rotation.

of material loss parameter (e.g., as in Fig. 3), these oscillations are not observed due to the large undamped reflection originating from the truncating $\mathrm{PEC}$, which is many orders higher than the reflection from the initial FS-A interface. This analysis indicates that, by choosing a large value of $\zeta_{0}$ typically in the range of $8 \omega$, one could opt for an absorber thickness smaller than $0.5 \lambda$. When the value of $\zeta_{0}$ is increased beyond a certain limit $(\approx 40 \omega)$, stability problems were encountered, which sets a maximum limit in choosing $\zeta_{0}$ for practical applications.

\section{RADiAL Finite-Volume MAXWELLIAN ABSORBER}

The concept of radially anisotropic cylindrical PML was reported in the framework of FDTD in [8]-[10] and for FETD applications in [11]. All these previous attempts in modeling cylindrical PML were motivated by representing Maxwell equations in the cylindrical coordinate system. Especially in the FDTD approach, in order to avoid staircasing errors at curved domain boundaries, curvilinear mesh arrangement were developed with update equations using the cylindrical coordinate system. On the contrary, within the framework of FVTD, unstructured meshing of the computational domain permits modeling of curved surfaces with a high level of accuracy and flexibility. In other words, the feature of the unstructured spatial discretization enables the FVTD method to model domain boundaries of any shape in a generalized manner. Consequently, there is no need for a special type of mesh arrangement like a curvilinear mesh and, thus, transforming the system update equations to a cylindrical coordinate system is not required. In the following, the theory of radial finite-volume absorbers is discussed in detail and a problem with two test scenarios in cylindrical geometry are simulated.

\section{A. Radial Anisotropy: Rotated Coordinate Analysis}

The direction of wave attenuation inside the absorber is given by the direction of anisotropy. In the previous discussion on the uniaxial material absorber model, the anisotropy direction was chosen along the $+x$-axis. In contrast, when considering a radial absorber model described in the cylindrical coordinate system $(\rho, \varphi, z)$, the anisotropy is defined in the radial direction $\rho$. In other words, as illustrated in Fig. 6, the local direction of anisotropy is along the $+x_{r}$-direction and the local coordinate $\left(x_{r}, y_{r}, z\right)$ can be viewed as a rotational transformation of the global coordinate $(x, y, z)$ through an angle $\varphi$ in the $x y$-plane. For the assumed $\mathrm{TE}_{z}$ formulation, the magnetic and electric field components in the rotated axes are given by 
$\boldsymbol{U}_{\boldsymbol{r}}=\left[H_{x_{r}}, H_{y_{r}}, E_{z}\right]^{T}$. As clearly noticed, the rotational transformation in the $x y$-plane has no effect on the electric field component $E_{z}$ along the $z$-axis. The field transformation from the global to the local axes is given by

$$
\left(\begin{array}{c}
H_{x_{r}} \\
H_{y_{r}} \\
E_{z}
\end{array}\right)=\overbrace{\left(\begin{array}{ccc}
\cos \varphi & \sin \varphi & 0 \\
-\sin \varphi & \cos \varphi & 0 \\
0 & 0 & 1
\end{array}\right)}^{\mathcal{R}}\left(\begin{array}{c}
H_{x} \\
H_{y} \\
E_{z}
\end{array}\right)
$$

where $\mathcal{R}$ denotes the rotation matrix defined by the angle of rotation $\varphi$. The system of equations in the local coordinate is written as [1]

$$
\begin{aligned}
\mu_{0} \partial_{t} H_{x_{r}} & =-\partial_{y_{r}} E_{z}-\mu_{0} K_{x_{r}} \\
\mu_{0} \partial_{t} H_{y_{r}} & =\partial_{x_{r}} E_{z}-\mu_{0} \zeta_{x_{r}} H_{y_{r}} \\
\varepsilon_{0} \partial_{t} E_{z} & =\partial_{x_{r}} H_{y_{r}}-\partial_{y_{r}} H_{x_{r}}-\varepsilon_{0} \zeta_{x_{r}} E_{z} \\
\mu_{0} \partial_{t} K_{x_{r}} & =\zeta_{x_{r}} \partial_{y_{r}} E_{z} .
\end{aligned}
$$

Apart from the standard field vectors defined in $\boldsymbol{U}_{\boldsymbol{r}}$, there is a scalar field given by $K_{x_{r}}$, which represents the magnetic counterpart of polarization current, as defined in [1] and [4]. The above set of update equations differ from that of the uniaxial absorber (8)-(11) with respect to the fourth scalar field $K_{x_{r}}$. Here, the update equation of the fourth field is represented as a partial differential equation (PDE) in space and time variables. In fact, one could also define the fourth field update equation as an ODE in time, as discussed in [1], but here, the usage of a PDE form of the update equation instead of an ODE ensures mathematical simplicity in modeling radial anisotropy. Numerically, once the flux function of $H_{x_{r}}$ is known, the flux function of $K_{x_{r}}$ is directly obtained from that of $H_{x_{r}}$ with an appropriate change of sign. In order to model the radial anisotropy using the field vectors $U$ and $K_{x}$ defined in the global coordinate $(x, y, z)$, all the local field quantities inside each control volume must be transformed back to the global coordinate system. This reverse transformation from the local to global coordinate system is achieved using the following relation:

$$
\left[x_{r}, y_{r}, z\right]^{T} \stackrel{\mathcal{R}^{-1}}{\Longrightarrow}[x, y, z]^{T} .
$$

Similarly, the field quantities in the local coordinate should be transformed to the global coordinate as follows:

$$
\left[H_{x_{r}}, H_{y_{r}}, E_{z}, K_{x_{r}}\right]^{T} \stackrel{\mathcal{R}^{-1}}{\Longrightarrow}\left[H_{x}, H_{y}, E_{z}, K_{x}\right]^{T} .
$$

For complete modeling of the radial anisotropy in the global coordinate, the partial derivatives $\left(\partial_{x_{r}}, \partial_{y_{r}}\right)$ present in (13)-(16) should be transformed back to the global coordinate using the chain rule as follows:

$$
\begin{aligned}
\partial_{x_{r}} & =\partial_{x_{r}}(x) \partial_{x}+\partial_{x_{r}}(y) \partial_{y} \\
\partial_{y_{r}} & =\partial_{y_{r}}(x) \partial_{x}+\partial_{y_{r}}(y) \partial_{y} .
\end{aligned}
$$

Substituting (17) in (19) and (20) results in the following:

$$
\begin{aligned}
& \partial_{x_{r}}=\cos \varphi \partial_{x}+\sin \varphi \partial_{y} \\
& \partial_{y_{r}}=-\sin \varphi \partial_{x}+\cos \varphi \partial_{y} .
\end{aligned}
$$

Using (21) and (22) in (18) and after some algebraic manipulations, all the field components inside the radial absorber can be expressed as follows:

$$
\begin{aligned}
\mu_{0} \partial_{t} H_{x}= & -\partial_{y} E_{z}+\zeta_{x_{r}} \mu_{0} H_{y} \cos \varphi \sin \varphi \\
& -\zeta_{x_{r}} \mu_{0} H_{x} \sin ^{2} \varphi-\mu_{0} K_{x_{r}} \cos \varphi \\
\mu_{0} \partial_{t} H_{y}= & \partial_{x} E_{z}+\zeta_{x_{r}} \mu_{0} H_{x} \cos \varphi \sin \varphi \\
& -\zeta_{x_{r}} \mu_{0} H_{y} \cos ^{2} \varphi-\mu_{0} K_{x_{r}} \sin \varphi \\
\varepsilon_{0} \partial_{t} E_{z}= & \partial_{x} H_{y}-\partial_{y} H_{x}-\zeta_{x_{r}} \varepsilon_{0} E_{z} \\
\mu_{0} \partial_{t} K_{x_{r}}= & -\zeta_{x_{r}} \sin \varphi \partial_{x} E_{z}+\zeta_{x_{r}} \cos \varphi \partial_{y} E_{z} .
\end{aligned}
$$

Comparing (13)-(15) with (23)-(25), it is noticed that the transformation from the local coordinate to the global coordinate does not change the structure of Maxwell equations, except for the lower order terms (terms with no spatial derivatives). This is a direct consequence of rotational invariance of the Maxwell system. Logically the fourth scalar field $K_{x_{r}}$ should also be split into two components in the $x$ - and $y$-directions. However, in the system of update (23)-(26), this fourth field can still be represented in the local coordinate system. It should be also noticed that the right-hand side of the fourth update (26) is represented in global coordinates. This mathematical manipulation is deliberate in order to use four instead of five update equations. There are clearly two extreme situations, which can be directly tested from the above update (23)-(26), namely, $\varphi=0$ and $\varphi=90^{\circ}$. These two situations yield update equations corresponding to uniaxial in the $+x$ - and $+y$-directions, respectively. Finally, the system of equations representing the radial absorber model is written using the flux-based FVTD update equations as follows:

$$
\begin{aligned}
\partial_{t} H_{x}= & \frac{-1}{\mu_{0}\left|V_{i}\right|} \sum_{k=1}^{f}\left(\mathcal{F}_{H_{x k}} \cdot \boldsymbol{n}_{k}\left|S_{k}\right|\right)-K_{x_{r}} \cos \varphi \\
& -\zeta_{x_{r}} H_{x} \sin ^{2} \varphi+\zeta_{x_{r}} H_{y} \cos \varphi \sin \varphi \\
\partial_{t} H_{y}= & \frac{-1}{\mu_{0}\left|V_{i}\right|} \sum_{k=1}^{f}\left(\mathcal{F}_{H_{y k}} \cdot \boldsymbol{n}_{k}\left|S_{k}\right|\right)-K_{x_{r}} \sin \varphi \\
& -\zeta_{x_{r}} H_{y} \cos ^{2} \varphi+\zeta_{x_{r}} H_{x} \cos \varphi \sin \varphi \\
\partial_{t} E_{z}= & \frac{-1}{\varepsilon_{0}\left|V_{i}\right|} \sum_{k=1}^{f}\left(\mathcal{F}_{E_{z k}} \cdot \boldsymbol{n}_{k}\left|S_{k}\right|\right)-\zeta_{x_{r}} E_{z} \\
\partial_{t} K_{x_{r}}= & \zeta_{x_{r}} \sin \varphi \frac{1}{\mu_{0}\left|V_{i}\right|} \sum_{k=1}^{f}\left(\mathcal{F}_{H_{y k}} \cdot \boldsymbol{n}_{k}\left|S_{k}\right|\right) \\
& +\zeta_{x_{r}} \cos \varphi \frac{1}{\mu_{0}\left|V_{i}\right|} \sum_{k=1}^{f}\left(\mathcal{F}_{H_{x k}} \cdot \boldsymbol{n}_{k}\left|S_{k}\right|\right) .
\end{aligned}
$$

Interestingly, the flux terms computed for (27) and (28) can be reused in (30) and this flux recycling helps in reducing computational effort.

\section{B. Numerical Experiments}

For testing the radial finite-volume absorber formulation, a test problem is chosen and, as before, two models (test and reference) are created (Fig. 7). For all the experiments carried out here, the thickness of the absorber $d_{\text {abs }}$ is fixed to $0.5 \lambda$ and 


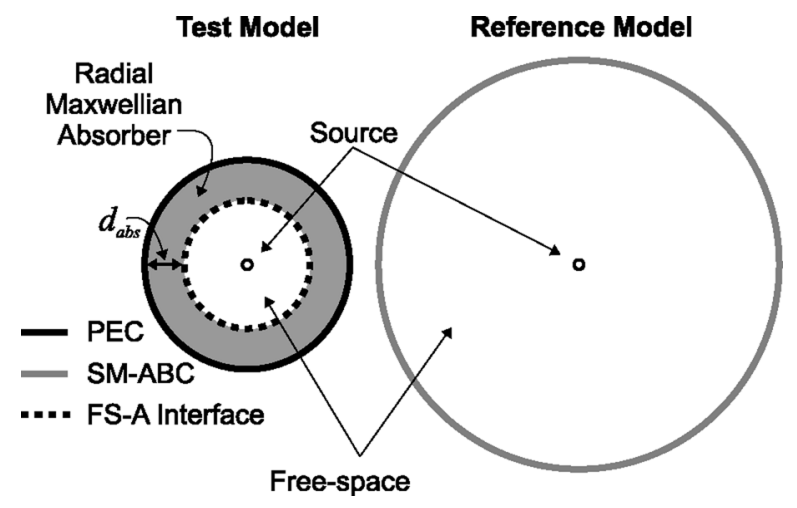

Fig. 7. Model problem illustrating the test and reference domains. The test model of radius of approximately $3.33 \lambda$ is truncated using the finite-volume Maxwellian absorber of thickness $d_{\mathrm{abs}}=0.5 \lambda$.

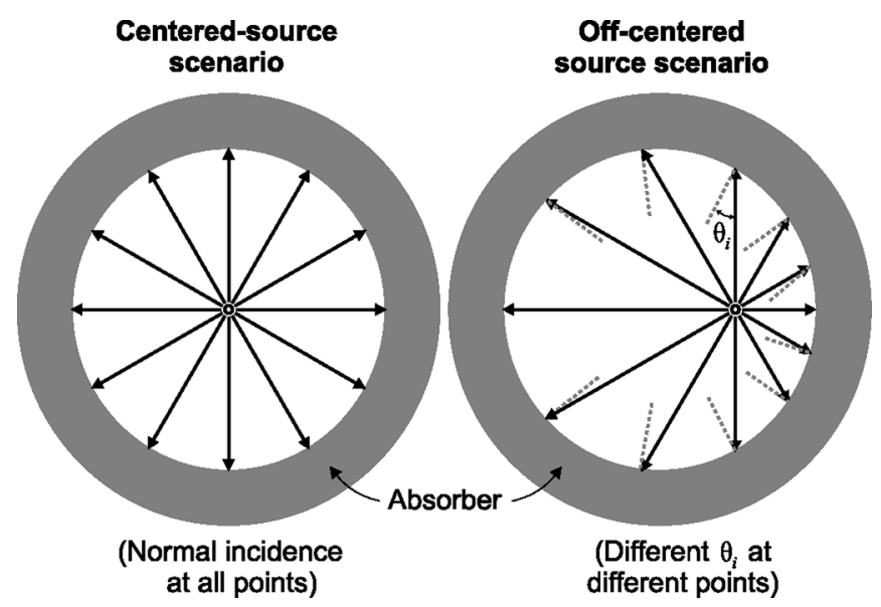

Fig. 8. Different angles of incidence at the FS-A interface based on source location.

the material loss parameter $\zeta_{0}$ is fixed to $8 \omega$. These values are chosen from the results of the uniaxial absorber model discussed before (refer to Fig. 5). The spatial discretization employed in the following problem corresponds to triangular cells with edge dimensions $\approx \lambda / 30$. Two representative numerical experiments were carried out as illustrated in Fig. 8. In the first example, the 2-D model of the cylindrical domain is excited with an axial line source placed exactly at the center of the cylindrical domain, as depicted on the left-hand side of Fig. 8. The electric field of the line source is impressed with a time-harmonic source function. Since the location of the line source is exactly at the center, the generated cylindrical wave will impinge on the FS-A interface at normal incidence, i.e., $\theta=0^{\circ}$ uniformly at all locations on the FS-A interface. The numerical error due to truncation using the radial absorber is computed from the difference in field values between the test and reference model. In the second experiment, the source location is shifted towards one side, as shown on the right-hand side of Fig. 8. In this case, a large range of incidence angles will be involved. The performance of the radial absorber is compared with the first-order accurate SM-ABC commonly used in FVTD simulations. The results of the first numerical experiment is shown in Fig. 9. Theoretically, at normal incidence, the SM-ABC is a perfect ABC. Numerically, it performs at its best and its accuracy is comparable to that of the finite-volume
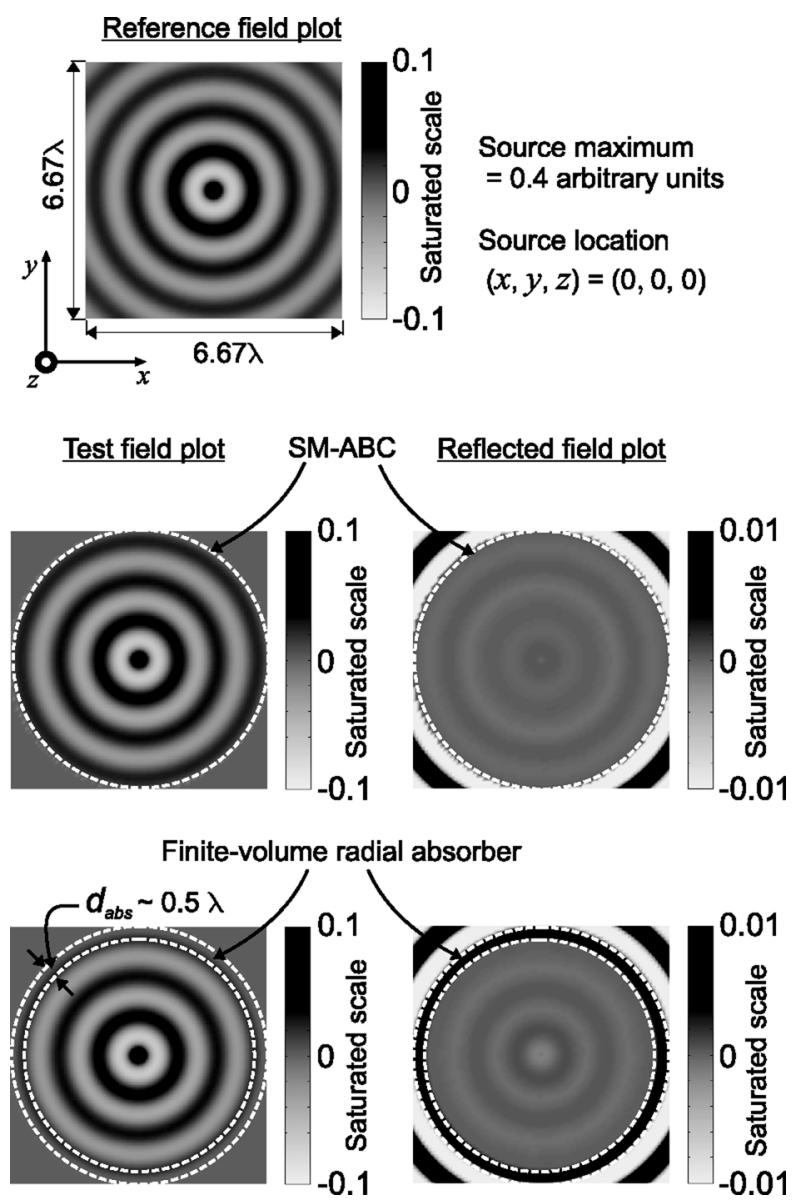

Fig. 9. Comparison of results of numerical error using SM-ABC and finitevolume Maxwellian absorber for the centered-source scenario.

radial absorber. The reflection at normal incidence in the case of the radial absorber is predominantly due to discretization errors inside the absorber. When the source location is displaced away from the center, a wide range of incidence angles comes into action and the performance of the SM-ABC degrades drastically. In this case, the performance of the finite-volume radial absorber is substantially better than SM-ABC and the numerical error remains stable for any source location (Fig. 10). The numerical reflection due to the finite-volume radial absorber computed at a test point is compared with that of the SM-ABC, and the results are shown in Fig. 11. As clearly observed, the performance of the finite-volume absorber model is approximately $10 \mathrm{~dB}$ better than the SM-ABC. The apparent degradation in the performance compared to the uniaxial model is not unexpected because it is due to multiple reflections at grazing angles of incidence. Nevertheless, the performance of the radial absorber confirms a significant improvement compared to the existing SM-ABC.

\section{Outlook for Extension to Three-Dimensional (3-D) Geometries}

The proposed radial absorber can be generalized to 3-D geometries in two different ways. The first option is to employ a spherical absorber model with the direction of anisotropy defined along the radial direction. In order to represent the update equations only using the global field values, one has to perform a 

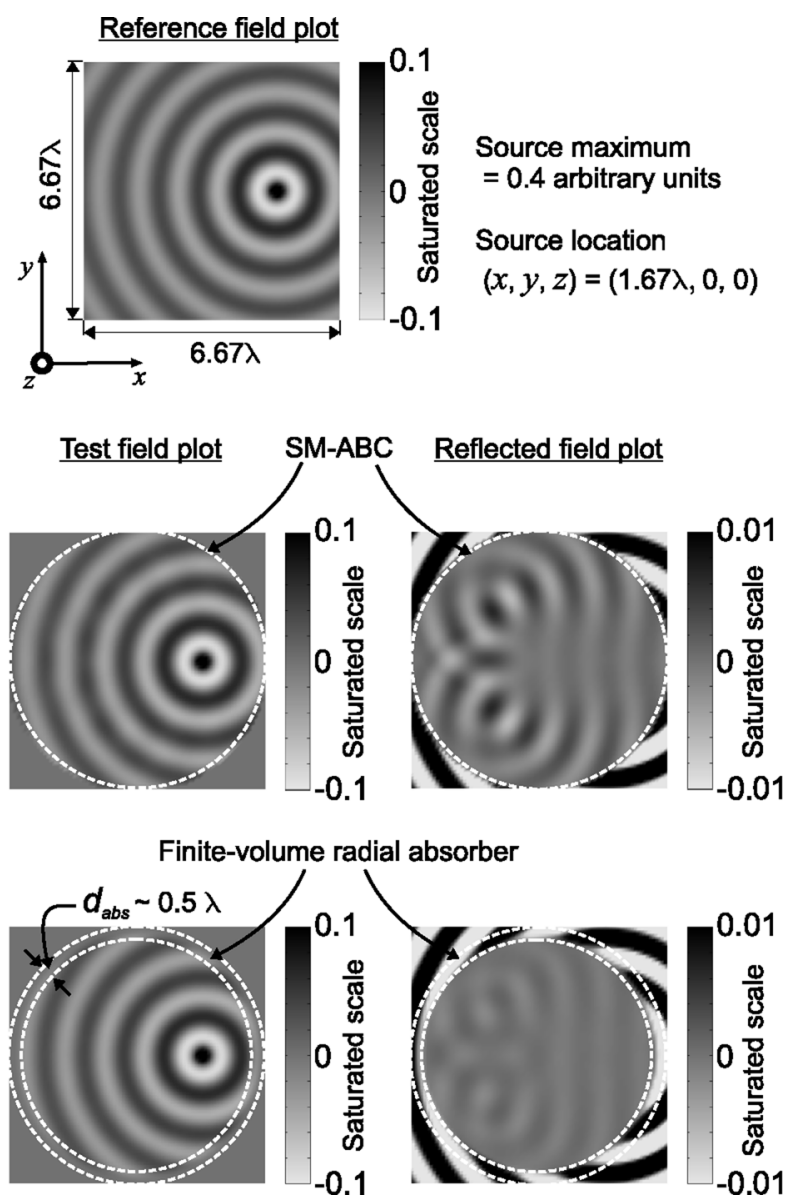

Fig. 10. Comparison of results of numerical error using SM-ABC and finitevolume Maxwellian absorber for the off-centered source scenario.

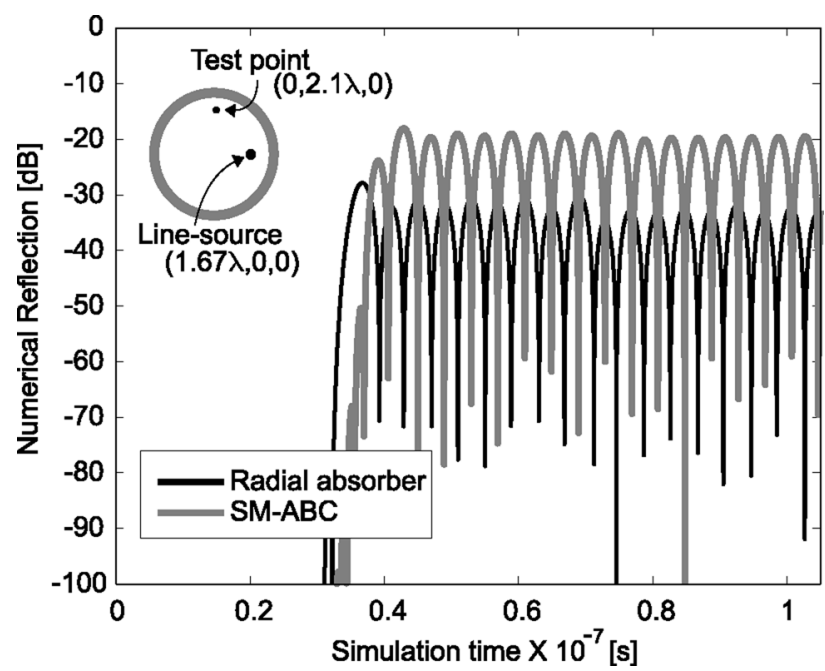

Fig. 11. Comparison of numerical reflection at a test point due to the finitevolume Maxwellian absorber and SM-ABC for the example scenario shown in the inset.

inverse transformation for obtaining a locally uniaxial and globally spherical absorber behavior. Contrary to the 2-D radial absorber case, the spherical transformation will affect all the field components due to two degrees of rotation involved.
However, for elongated structures, employing a spherical absorber for domain truncation will drastically increase the computational volume. Hence, to avoid the computational overhead, a second option is proposed employing a cylindrical absorber model with two hemispherical absorbers appended as the top and bottom covers. Without loss of generality, if the $z$-axis is assumed to align along the longer dimension of the elongated structure, then a cylindrical absorber can be employed with the $z$-axis chosen as the axis of the absorber. Appending spherical absorbers on the top and bottom of the cylindrical absorber avoids corner regions and, hence, offers more accurate domain truncation. The proposed idea for 3-D extension is currently under further investigation and will be a topic of interest in the future.

\section{CONCLuSions}

A new model for mesh truncation using uniaxial Maxwellian absorber in the framework of FVTD was introduced. The performance of the uniaxial absorber was tested for angular response using a coarse spatial discretization with around 12 points per wavelength. For a variation of the angle of incidence from near normal to $50^{\circ}$, the reflection coefficient of the FVTD Maxwellian absorber was lower than $-40 \mathrm{~dB}$, demonstrating the good performance achieved in practice for such coarse spatial discretization. The accuracy of the proposed model was found to further increase when employed on meshes with spatial discretization with approximately 20 points per wavelength. Numerical reflection coefficients in the range of $-60 \mathrm{~dB}$ were achieved on fine meshes confirming convergence of numerical results. The uniaxial finite-volume Maxwellian absorber was further extended for modeling cylindrical geometries. The modified formulation of the radial absorber adapts naturally to the FVTD algorithm without any need for coordinate transformation, convolution, or curvilinear meshing. The performance of the radial absorber was compared with the existing SM-ABC. The overall performance of the radial absorber was found to be significantly better than the existing mesh truncation technique in the framework of the FVTD method.

\section{REFERENCES}

[1] K. Sankaran, C. Fumeaux, and R. Vahldieck, "Finite-volume Maxwellian absorber on unstructured grid," in IEEE MTT-S Int. Microw. Symp. Dig., Jun. 2006, pp. 169-172.

[2] R. W. Ziolkowski, "The design of Maxwellian absorbers for numerical boundary conditions and for practical applications using engineered artificial materials," IEEE Trans. Antennas Propag., vol. 45, no. 4, pp. 656-671, Apr. 1997.

[3] — , "Time-derivative Lorentz materials and their utilization as electromagnetic absorbers," Phys. Rev. E, Stat. Phys. Plasmas Fluids Relat. Interdiscip. Top., vol. 55, no. 6, pp. 7696-7703, Jun. 1997.

[4] — "Time-derivative Lorentz material model-based absorbing boundary condition," IEEE Trans. Antennas Propag., vol. 45, no. 10, pp. 1530-1535, Oct. 1997.

[5] A. Roden and S. Gedney, "Convolution PML (CPML): An efficient FDTD implementation of the CFS-PML for arbitrary media," Microw. Opt. Technol. Lett., vol. 27, no. 5, pp. 334-339, 2000.

[6] J.-P. Bérenger, "A perfectly matched layer for the absorption of electromagnetic waves," J. Comput. Phys., vol. 114, no. 2, pp. 185-200, 1994.

[7] — , "Three-dimensional perfectly matched layer for the absorption of electromagnetic waves," J. Comput. Phys., vol. 127, no. 2, pp. 363-379, Sep. 1996. 
[8] F. Collino and P. Monk, "The perfectly matched layer in curvilinear coordinates," SIAM J. Sci. Comput., vol. 19, pp. 2061-2090, Nov. 1998.

[9] F. L. Teixeira and W. C. Chew, "Perfectly matched layer in cylindrical coordinates," in IEEE AP-S Int. Symp., Jul. 1997, vol. 3, pp. 1908-1911.

[10] P. G. Petropoulos, "Reflectionless sponge layers as absorbing boundary conditions for the numerical solution of Maxwell equations in rectilinear, cylindrical, and spherical coordinates," SIAM J. Appl. Math., vol. 60, pp. 1037-1058, Feb.-March 2000.

[11] M. Kuzuoğlu and R. Mittra, "Investigation of nonplanar perfectly matched absorbers for finite-element mesh truncation," IEEE Trans. Antennas Propag., vol. 45, no. 3, pp. 474-486, Mar. 1997.

[12] N. K. Madsen and R. W. Ziolkowski, "A three-dimensional modified finite volume technique for Maxwell's equations," Electromagnetics, vol. 10 , pp. 147-161, 1990.

[13] V. Shankar, A. H. Mohammadian, and W. F. Hall, "A time-domain, finite-volume treatment for the Maxwell equations," Electromagnetics, vol. 10, pp. 127-145, 1990.

[14] P. Bonnet, X. Ferrieres, B. Michielsen, P. Klotz, and J. Roumiguiéres, Time Domain Electromagnetics, S. M. Rao, Ed. New York: Academic, 1997, ch. 9, pp. 307-367.

[15] C. Fumeaux, D. Baumann, P. Leuchtmann, and R. Vahldieck, "A generalized local time-step scheme for efficient FVTD simulations in strongly inhomogeneous meshes," IEEE Trans. Microw. Theory Tech., vol. 52, no. 3, pp. 1067-1076, Mar. 2004.

[16] S. Abarbanel and D. Gottlieb, "On the construction and analysis of absorbing layers in CEM," Appl. Numer. Math., no. 27, pp. 331-340, 1998.

[17] K. Sankaran, C. Fumeaux, and R. Vahldieck, "Cell-centered finite-volume based perfectly matched layer for time domain maxwell system," IEEE Trans. Microw. Theory Tech., vol. 54, no. 3, pp. 1269-1276, Mar. 2006.

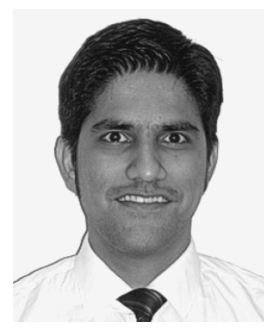

Krishnaswamy Sankaran (S'98) received the B.Eng. degree (with a first-class distinction) in electrical and electronics engineering from the University of Madras, Madras, India, in 2002, the M.Sc. degree in information and communication engineering from the University of Karlsruhe $\mathrm{TH}$, Karlsruhe, Germany, in 2004, and is currently working toward the Ph.D. degree from the Swiss Federal Institute of Technology (ETH) Zürich, Zürich, Switzerland.

From October 2003 to May 2004, he was a Research Trainee with the European Commission, Joint Research Centre, Ispra, Italy, where he was involved in the field of radar systems engineering and remote sensing. In June 2004, he joined the ETH Zürich, where he is currently with the Laboratory for Electromagnetic Field Theory and Microwave Electronics (IFH). His main research interests are numerical methods for solving EM field problems, computational physics, and applied mathematics.

Mr. Sankaran is currently vice-chair of the IEEE Student Branch Zürich. He was the recipient of a full postgraduate scholarship and he was one of the recipients of the 2006 Best Student Paper Award presented at the IEEE Microwave Theory and Techniques Society (IEEE MTT-S) International Microwave Symposium (IMS), San Francisco, CA.

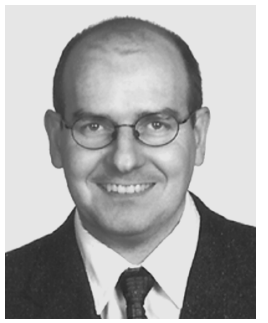

Christophe Fumeaux (M'03) received the Diploma and Ph.D. degrees in physics from the Swiss Federal Institute of Technology (ETH) Zürich, Switzerland, in 1992 and 1997, respectively.

From 1998 to 2000, he was a Post-Doctoral Researcher involved in infrared technology with the School of Optics, University of Central Florida, Orlando. In 2000, he joined the Swiss Federal Office of Metrology, Bern, Switzerland, as a Scientific Staff Member. Since 2001, he has been a Research Associate with the Laboratory for Electromagnetic Fields and Microwave Electronics (IFH), ETH Zürich, Switzerland. During Fall 2005, he was a Visiting Scientist with the Laboratory of Sciences and Materials for Electronics, and of automatic (LASMEA), University of Blaise Pascal, Clermont-Ferrand, France. His current main research interest concerns computational electromagnetics in the time domain for numerical analysis of microwave circuits and antennas.

Dr. Fumeaux has been the chairman of the IEEE Swiss Joint Chapter on Microwave Theory and Techniques, Antennas and Propagation, and Electromagnetic Compatibility (EMC) since January 2006. He was the recipient of the ETH Silver Medal of Excellence for his doctoral dissertation. He was the corecipient of the 2004 Applied Computational Electromagnetics Society (ACES) Outstanding Paper Award.

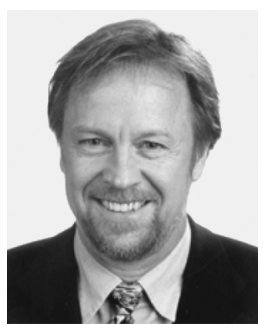

Rüdiger Vahldieck (M'85-SM'86-F'99) received the Dipl.-Ing. and Dr.-Ing. degrees in electrical engineering from the University of Bremen, Bremen, Germany, in 1980 and 1983, respectively.

From 1984 to 1986, he was a Post-Doctoral Fellow with the University of Ottawa, Ottawa, ON, Canada. In 1986, he joined the Department of Electrical and Computer Engineering, University of Victoria, Victoria, BC, Canada, where he became a Full Professor in 1991. During the fall of 1992 and the spring of 1993, he was a Visiting Scientist with the FerdinandBraun-Institute für Hochfrequenztechnik, Berlin, Germany. In 1997, he became a Professor of EM-field theory with the Swiss Federal Institute of Technology (ETH) Zürich, Zürich, Switzerland, and Head of the Laboratory for Electromagnetic Fields and Microwave Electronics (IFH) in 2003. His research interests include computational electromagnetics in the general area of electromagnetic compatibility (EMC) and, in particular, for computer-aided design of microwave, millimeter-wave, and opto-electronic integrated circuits. Since 1981, he has authored or coauthored over 300 technical papers in books, journals, and conferences, mainly in the field of microwave computer-aided design.

Prof. Vahldieck is the past president of the IEEE 2000 International Zürich Seminar on Broadband Communications (IZS2000). Since 2003, he has been president and general chairman of the International Zürich Symposium on Electromagnetic Compatibility. He is a member of the Editorial Board of the IEEE TRANSACTIONS ON MicrowaVe THEORY AND TeChNIQUes. From 2000 to 2003, he was an associate editor for the IEEE MiCROWAVE AND WIRELESS COMPONENTS LETTERS, and from July 2003 until the end of 2005, he was the editor-in-chief. Since 1992, he has served on the Technical Program Committee (TPC) of the IEEE Microwave Theory and Techniques Society (IEEE MTT-S) International Microwave Symposium (IMS), the IEEE MTT-S Technical Committee on Microwave Field Theory, and in 1999, on the TPC of the European Microwave Conference. From 1998 to 2003, he was the chapter chairman of the IEEE Swiss Joint Chapter on Microwave Theory and Techniques, Antennas and Propagation, and EMC. Since 2005, he has been president of the Swiss Research Foundation on Mobile Communications. He was the recipient of the J. K. Mitra Award of the Institution of Electronics and Telecommunication Engineers (IETE) (in 1996) for the best research paper in 1995 and was corecipient of the Outstanding Publication Award of the Institution of Electronic and Radio Engineers in 1983. He was the corecipient of the 2004 Applied Computational Electromagnetic Society (ACES) Outstanding Paper Award. 